\title{
Optimal control of aeroacoustic noise generated by cylinder vortex interaction
}

\author{
S.S. Collis ${ }^{1}$, K. Ghayour ${ }^{2}$ and M. Heinkenschloss ${ }^{3}$ \\ ${ }^{1}$ Mechanical Engineering and Materials Science, Rice University, 6100 Main Street, \\ MS 321, Houston, TX 77005, USA. collis@rice.edu \\ ${ }^{2}$ Computational and Applied Mathematics and Mechanical Engineering and Materials Science, \\ Rice University, 6100 Main Street, MS 134, Houston, TX 77005, USA. kghayour@caam.rice.edu \\ ${ }^{3}$ Computational and Applied Mathematics, Rice University, 6100 Main Street, \\ MS 134, Houston, TX 77005, USA. heinken@rice.edu
}

\begin{abstract}
This paper presents an optimal control formulation and solution for an idealized Blade Vortex Interaction (BVI) problem. This problem consists of the interaction of an inviscid vortex pair with a circular cylinder in a steady Mach 0.3 uniform flow with wall-normal velocity used as control on the cylinder surface. This model problem captures the fundamental noise generation process of the BVI phenomena while mitigating many of the complexities of the full rotorcraft problem. The optimal control problem is solved using a gradient based method where gradient information is computed from a continuous adjoint analysis of the governing unsteady Euler equations. The BVI wave packet is targeted by defining an objective function that measures the square amplitude of pressure fluctuations in an observation region over a time interval of interest. When the observation region encloses the entire flow a $6 \mathrm{db}$ reduction in overall BVI noise is obtained. When the observation region is limited to the region of greatest BVI noise, a $13 \mathrm{db}$ reduction is obtained. The optimal control, unlike most common mitigation methods, does not target the interaction directly — instead, the computed boundary control modulates the potential flow about the cylinder, producing a wave packet of the correct amplitude and phase to approximately cancel the BVI noise in the observation region.
\end{abstract}

\section{INTRODUCTION}

Under normal flight conditions, the wake and trailing tip vortices from the blades of rotorcraft naturally convect downward and away from the rotor disk. However, for certain low speed descending flight paths, the trailing vortices can pass close to the rotor blades leading to a phenomenon referred to as Blade-Vortex Interaction (BVI). As the trailing vortex interacts with the rotor blade, a large fluctuation in loading occurs near the leading edge of the blade resulting in a strong dipole type acoustic source. The noise generated by BVI is particularly bothersome since BVI commonly occurs near the ground as a rotorcraft slows and descends for landing and the sound generated by 
BVI is directed forward and downward at approximately $35^{\circ}$ with respect to the rotor plane. Thus BVI noise can be very severe, leading to environmental noise pollution in commercial applications and increased observability for military applications.

Extensive research, both experimental and computational, has been performed to study the fluid dynamics and acoustic mechanisms that result from BVI as well as to validate predictive techniques (see e.g. Krishnamoorthy and Marshall (1998); McCluer (1996); JanakiRam (1990); Caradonna et al. (1988)). These studies have improved the basic understanding of rotor-blade vortex interaction noise and have indicated various methods for controlling this phenomena. In particular, experimental studies have shown that the intensity of BVI noise depends on many factors such as tip vortex strength and structure, distance between the vortex and the subsequent blade, and the local geometry of the interaction. Based on these observations, three major mitigation methods are currently pursued (Gervais, 2001): 1) Reducing the strength of the shed vortices or increasing the core size of the vortices, 2) Designing and controlling the rotor blades to effectively weaken the impulsive load response of the blade, and 3) Increasing the miss-distance between the rotor tip-path plane and the shed vortex. Various control strategies have been investigated to achieve one or more of these objectives including: Flight path control, passive leading-edge suction, passive blade design, and active rotor control (see e.g. Schmitz (1998); Baeder (1997); Lee (1994); Yu et al. (1997)). Active rotor control can take several forms including higher harmonic pitch control, individual blade control, and on-blade control (see Yu et al. (1997) for a review).

In general, the control of a complex physical process like BVI requires a thorough understanding of the underlying physics. Unfortunately, due to the complexity of BVI, prior research on noise control has largely been through trial and error applications of various control strategies based on physical intuition. One of the main approaches to better understand the physics of the acoustic triggering process in BVI noise radiation is the "model building" approach (Schmitz, 1998; Sim and Schmitz, 2000) in which a simplified model of BVI noise is proposed and flight variables that can be used to reduce the BVI noise are explored. While this model building approach is useful to gain an understanding of the physics, it is relatively less useful in devising control strategies since the underlying physics may be intimately coupled so that controlling one aspect of the BVI process may negatively impact another. To avoid this, we take a different approach in which optimal control theory is used along with a high fidelity computational fluid dynamics simulation to devise controls that are aware of the full physics of the BVI generation process. The success of this approach relies on the fidelity of the mathematical model of the flow physics, the accuracy of numerical computations, and the choice of a suitable control mechanism.

In Collis et al. (2001a) and Collis et al. (2001b) we laid the foundation for optimal control of flows governed by the unsteady compressible Navier-Stokes equations. Building upon the experience and knowledge gained from these studies, in this paper, we focus on controlling the noise generated by a two-dimensional model BVI problem consisting of the interaction of an inviscid vortex pair with a circular cylinder in a steady Mach 0.3 uniform flow. This model problem captures the fundamental noise 
generation process of the BVI phenomena while mitigating many of the complexities of the full rotorcraft problem. As such, it serves as a stepping stone towards optimal control of realistic BVI flows.

Motivated by the development of small-scale surface mounted actuators and sensors and the success of these devices in other flow control contexts (McMichael, 1996; Gadel-Hak, 2000), we depart from the prior control approaches described above, and instead take as our control mechanism the time- and space-dependent distribution of surface normal velocity (suction and blowing). In the results section, it is demonstrated that the obtained optimal distribution of this control mechanism reduces BVI noise significantly despite the fact that the miss-distance remains virtually unaltered by the control actuation. Because optimal control has full knowledge of the governing equations (state equations) and the impact of boundary control actuation on flow variables (both mean and acoustic) in the domain, it can use the full flow physics to predict a control actuation on the blade surface that effectively cancels BVI noise in the observation region. This aspect of the work will be discussed in the results section.

The paper begins with a description of the problem formulation including the state equations and boundary conditions as well as a discussion of our solution approach. We then describe in detail the idealized BVI model problem investigated here. Results are presented for two optimal control test cases that target different aspects of the BVI sound signature and the paper ends with a discussion of the results and conclusions.

\section{PROBLEM FORMULATION AND NUMERICAL SOLUTION}

\subsection{State Equations}

Let $\Omega \subset \mathbb{R}^{2}$ denote the spatial domain occupied by the fluid and let $\Gamma$ denote its spatial boundary. By $\mathbf{u}=\left(\rho, v_{1}, v_{2}, T\right)^{T}$ we denote the primitive flow variables, where $\rho(t, \mathbf{x})$ is the density, $v_{i}(t, \mathbf{x})$ denotes the velocity in $x_{i}$-direction, $i=1,2, \mathbf{v}=\left(v_{1}, v_{2}\right)^{T}$, and $T(t, \mathbf{x})$ denotes the temperature. The pressure $p$ and the total energy per unit mass $E$ are given by

$$
p=\frac{\rho T}{\gamma \mathrm{M}^{2}}, \quad E=\frac{T}{\gamma(\gamma-1) \mathrm{M}^{2}}+\frac{1}{2} \mathbf{v}^{T} \mathbf{v},
$$

respectively, where $\gamma$ is the ratio of specific heats and $\mathrm{M}$ is the reference Mach number. We write the conserved variables as functions of the primitive variables,

$$
\mathbf{q}(\mathbf{u})=\left(\rho, \rho v_{1}, \rho v_{2}, \rho E\right)^{T}
$$

and we define the inviscid flux vectors

$$
\mathbf{F}^{1}(\mathbf{u})=\left(\begin{array}{c}
\rho v_{1} \\
\rho v_{1}^{2}+p \\
\rho v_{2} v_{1} \\
(\rho E+p) v_{1}
\end{array}\right) \quad \text { and } \quad \mathbf{F}^{2}(\mathbf{u})=\left(\begin{array}{c}
\rho v_{2} \\
\rho v_{1} v_{2} \\
\rho v_{2}^{2}+p \\
(\rho E+p) v_{2}
\end{array}\right)
$$


The two-dimensional compressible Euler equations for the time interval $\left[t_{0}, t_{f}\right]$ can now be written as

$$
\mathbf{q}(\mathbf{u})_{t}+\mathbf{F}^{1}(\mathbf{u})_{x_{1}}+\mathbf{F}^{2}(\mathbf{u})_{x_{2}}=\mathbf{0} \quad \text { in }\left(t_{0}, t_{f}\right) \times \Omega,
$$

with boundary conditions

$$
\mathbf{B}(\mathbf{u} ; \nabla \mathbf{u} ; g)=\mathbf{0} \quad \text { on }\left(t_{0}, t_{f}\right) \times \Gamma,
$$

and initial conditions

$$
\mathbf{u}\left(t_{0}, \mathrm{x}\right)=\mathbf{u}_{0}(\mathrm{x}) \quad \text { in } \Omega,
$$

In the boundary conditions [4], the function $g$ is the control which is taken to be suction and blowing in the wall normal direction on a subset $\Gamma_{c}$ of the boundary $\Gamma$. The precise form of the boundary conditions [4] used in our computations will be specified next.

A nonstandard and nontrivial task that arises in our application is the formulation and implementation of boundary conditions. For the Euler equations the number of physical boundary conditions that need to be imposed at boundary surfaces depends on the signs of the eigenvalues $\mathbf{v}^{T} \mathbf{n}, \mathbf{v}^{T} \mathbf{n}, \mathbf{v}^{T} \mathbf{n}+c$ and $\mathbf{v}^{T} \mathbf{n}-c$ of the matrix $n_{1} \partial \mathbf{F}_{1} / \partial \mathbf{q}+$ $n_{2} \partial \mathbf{F}_{2} / \partial \mathbf{q}$. Here $\mathbf{v}$ and $c$ are the local velocity and speed of sound respectively, $\mathbf{n}=\left(n_{1}\right.$, $\left.n_{2}\right)^{T}$ is the unit outward normal to the boundary, and $\partial \mathbf{F}_{i} / \partial \mathbf{q}$ represents the flux Jacobians with respect to the conservative variables. Our control is wall-normal suction and blowing on part of the boundary, which is modeled by $\mathbf{v}^{T} \mathbf{n}=g$. Thus the signs of the above mentioned eigenvalues depend on the value of the control. Since the control is allowed to vary over the actuated spatial boundary $\Gamma_{c}$ and in time, the signs of the eigenvalues may change in space and time. Accordingly, we have to dynamically adjust the number of physical boundary conditions imposed.

When one of the eigenvalues $\mathbf{v}^{T} \mathbf{n}, \mathbf{v}^{T} \mathbf{n}, \mathbf{v}^{T} \mathbf{n}+c$ and $\mathbf{v}^{T} \mathbf{n}-\mathrm{c}$ is negative, the associated characteristic propagates from the boundary towards the interior of the domain and a physical boundary condition must be imposed. On the other hand, a positive eigenvalue indicates flow of information from the inner domain towards the boundary, necessitating the imposition of a numerical boundary condition. Therefore, for subsonic suction $0<\mathbf{v}^{T} \mathbf{n}=g<c$ one physical and three numerical boundary conditions must be imposed, while for subsonic blowing $-c<\mathbf{v}^{T} \mathbf{n}=g<0$ three physical and one numerical boundary conditions are needed. For suction, the normal velocity $\mathbf{v}^{T} \mathbf{n}$ is the obvious flow quantity of choice. Numerous combinations of three flow quantities can potentially constitute the boundary condition for blowing. In our numerical experimentation we have had success imposing normal velocity, entropy $S$, and vorticity $\omega$. To summarize, for an impermeable wall or suction $\left(\mathbf{v}^{T} \mathbf{n} \geq 0\right)$ we impose the physical boundary condition

$$
\mathbf{v}^{T} \mathbf{n}-g=0
$$


whereas for blowing $\left(\mathbf{v}^{T} \mathbf{n}<0\right)$ we impose

$$
\mathbf{v}^{T} \mathbf{n}=g, \quad \ln \left(\frac{T^{1 /(\gamma-1)}}{\rho}\right)=S_{0}, \quad \partial_{x_{2}} v_{1}-\partial_{x_{1}} v_{2}=\omega_{0} .
$$

In this study, we set $\omega_{0}=S_{0}=0$, i.e., we force the injected fluid to be isentropic and irrotational.

We may combine [6] and [7] into

$$
\mathbf{B}(\mathbf{u}, \nabla \mathbf{u}, \mathbf{g}) \stackrel{\text { def }}{=}\left|\begin{array}{c}
\mathbf{v}^{T} \mathbf{n}-g \\
\left(\ln \left(\frac{T^{1 /(\gamma-1)}}{\rho}\right)-S_{0}\right) \min \{g, 0\}^{2} \\
\left(\partial_{x_{2}} v_{1}-\partial_{x_{1}} v_{2}-\omega_{0}\right) \min \{g, 0\}^{2}
\end{array}\right|=0
$$

Although we have used these boundary conditions successfully in several optimal control applications (see Collis et al. (2002) and Section 3), mathematical wellposedness of the compressible Euler equations with these boundary conditions has not yet been established and our success with these boundary conditions may be dependent on their implementation, which is discussed below.

\subsection{The Optimal Control Problem}

We are primarily interested in reducing the amplitude of sound waves in some region $\Omega_{\text {obs }}$ of the domain $\Omega$. Therefore we use

$$
\frac{1}{2} \int_{t_{0}}^{t_{f}} \int_{\Omega_{\mathrm{obs}}}\left(p-p_{a}\right)^{2} d \mathbf{x} d t
$$

as a measure of aeroacoustic noise to be reduced, where $p_{a}$ is the ambient or steady mean-flow pressure distribution. However, to arrive at a well-posed optimal control problem we also need to constrain the control input and its derivatives. We accomplish this by adding a suitable penalty term to [8]. Motivated by our experiences (Collis et al., 2001b) with boundary control of compressible Navier-Stokes equations, we solve

$$
\min _{g \in \mathcal{G}_{a d}} \quad J(g)
$$

where

$$
\begin{gathered}
J(g) \stackrel{\text { def }}{=} \int_{t_{0}}^{t_{f}} \int_{\Omega_{\mathrm{obs}}} \frac{\alpha_{0}}{2}\left(p-p_{a}\right)^{2} d \mathbf{x} d t \\
+\int_{t_{0}}^{t_{f}} \int_{\Gamma_{c}}\left(\frac{\alpha_{1}}{2}\left\|g_{t}\right\|_{2}^{2}+\frac{\alpha_{2}}{2}\|g\|_{2}^{2}+\frac{\alpha_{3}}{2}\|\nabla g\|_{2}^{2}+\frac{\alpha_{4}}{2}\|\Delta g\|_{2}^{2}\right) d \mathbf{x} d t,
\end{gathered}
$$


$\mathcal{G}_{a d}$ is the set of admissible controls, and $\alpha_{0}, \ldots, \alpha_{4}>0$ are weighting parameters. Furthermore, $\nabla g$ is the gradient of $g$ on the boundary, and $\Delta g$ is the Laplacian of $g$ on the boundary. For a flat boundary $\Gamma_{c}=\left\{\left(x_{1}, x_{2}\right) \in \mathbb{R}^{2}: x_{2}=0\right\}$ these are just $g_{x_{1}}$ and $g_{x_{1}, x_{1}}$, respectively. The set $\mathcal{G}_{a d}$ of admissible controls enforces the conditions $g(t, \mathbf{x})=$ $\nabla g(t, \mathbf{x})=0$ for $t \in\left(t_{0}, t_{f}\right)$ and $\mathbf{x} \in \partial \Gamma_{c}$ as well as $g\left(t_{0}, \mathbf{x}\right)=0, \mathbf{x} \in \Gamma_{c}$. The first two conditions ensure that the control goes smoothly to zero at the endpoints of the controlled boundary, i.e, at $\partial \Gamma_{c}$. The third condition enforces compatibility between the initial velocity field and boundary data.

\subsection{Gradient Computations Using Adjoint Equations}

Since our control varies over part of the boundary and in time, the number of control variables obtained after discretization is large (in our computations the number of control variables is of the order $10^{5}$ ). Therefore we use a gradient based optimization algorithm for the solution of the discretization of [9]. We use a nonlinear conjugate gradient method (Nocedal and Wright, 1999), but the specific choice of the optimization algorithm is not crucial for the purpose of this paper. Because of the large number of controls, the gradients are computed using the adjoint equation approach. This approach is widely used. The papers by Abergel and Temam (1990); Berggren (1998); Collis et al. (2001b,a); He et al. (2000); Fursikov et al. (1998); Gunzburger and Manservisi (2000); He et al. (1997) represent a small sample of the work on adjoint methods for the optimization of unsteady flows. For the computations in this paper we use the optimize-then-discretize approach, i.e., we first derive the adjoint partial differential equations and corresponding boundary conditions and then discretize these. While the adjoint computations lead to several interesting and important mathematical, numerical, and implementational issues, these are beyond the scope of this paper. We refer to our earlier work Collis et al. (2001b) and to Collis et al. (2002) where more details may be found.

\subsection{Discretization}

The success of an optimal control approach for the BVI problem pivots around the accuracy of the acoustic field prediction that ultimately affects the accuracy of the gradient for updating the control. Acoustic waves are non-dissipative and nondispersive. Thus, high-order accurate numerical schemes with minimal dissipation and dispersion errors are essential for computational aeroacoustics. Our state solution is accomplished using an extension of the explicit finite-difference method described by Collis (1997). The Euler equations are formulated in a generalized coordinate system where the physical domain is mapped to a computational space; a unit square divided into an equally spaced grid system. This transformation allows clustering of grid points in regions of high gradients, simplifies the implementation of the boundary conditions, and allows the code to be used for moderately complex geometries. For this study spatial derivatives are approximated using standard sixth-order accurate central differences in the interior with third-order biased and one-sided differences at boundaries that are designed to enhance stability when used with explicit time advancement methods (Carpenter et al., 1993). To suppress the growth of high- 
wavenumber error modes, a fourth-order artificial dissipation term is added to the right hand side of the discretized equations. This dissipation term is computed using fourthorder accurate finite-differences and the dissipation parameter, $\epsilon$, is chosen to damp out the error modes while avoiding excessive dissipation in the resolved scales as established through numerical experimentation. Sponge terms (Israeli and Orszag, 1981; Collis, 1997) are used in the vicinity of the farfield boundary with a onedimensional Riemann invariant treatment at the farfield boundary (Collis, 1997). Incorporation of the suction/blowing boundary conditions into the state simulation poses a significant problem for high-order essentially non-dissipative discretizations. Our implementation of these boundary conditions is based on the work by Sesterhenn (2001). Details of our implementation may be found in Collis et al. (2002).

\section{RESULTS}

This section begins with a description of our model BVI problem followed by a brief description of the uncontrolled flow. Results are then presented for two optimal control problems that target different aspects of the BVI noise signature. The section closes with a discussion of the results.

\subsection{Problem Set-Up}

Given the computational expense of combining optimization with accurate aeroacoustics simulation, we have made a number of approximations and idealizations to make this work tractable. In particular, we use a two-dimensional model of BVI interaction to avoid performing optimal control on full three-dimensional simulations, which individually would tax current computational resources. Fortunately, the analytical work of Widnall (1971) indicates that the intensity of BVI noise is a function of the angle between the vortex tube axis and the blade span at the moment of interaction and the most intense interaction occurs when these two are parallel. Therefore, it is useful to study two-dimensional BVI problems. Furthermore, Nakamura (1981) points out the importance of the leading-edge of the rotor blade in the interaction process. As most blades have a blunt leading edge, we use a circular cylinder to model the leading edge region of a rotor blade. Finally, to further contain computational expense, we assume that the flow is symmetric about the horizontal axis. Thus, we model the BVI process as a parallel interaction of a vortex pair with a circular cylinder as shown schematically in Figure 1. Accurate simulation of a BVI event for a rotorcraft in flight may also include transonic effects, dynamic lift effects due to the pitching rotor blade, and three-dimensional effects and we do not consider these here.

In the following, the free stream velocity and the radius of the cylinder $R_{c}$ are used for nondimensionalization and the center of the cylinder is the origin of the coordinate system. The computational domain, $\Omega$, is the upper half of the annular region bounded by the cylinder surface at $r=1$, the far-field boundary at $r=15$, and the symmetry line $y=0$ as shown in Figure 1. At time $t=0$, a relatively strong inviscid vortex (Shu, 1997) of circulation -0.1875 and core radius $R_{v}=0.375$ is superimposed on the uniform flow at location $(-10,1)$ upstream of the cylinder. The core radius $R_{v}$ is defined as the radial position, relative to the center of the vortex, at which the velocity induced by the vortex 
attains its peak value. The vortex is advected downstream under the combined effect of its image vortex and the free stream. It loses about 60 percent of its strength because of poor azimuthal resolution of the mesh far away from the center of the cylinder. In the meantime, the initial transient associated with the superposition is reflected and scattered by the cylinder and it takes some time for this transient to leave the domain. As the vortex is advected downstream, the mesh resolution in the tangential direction improves linearly with the radial position $r$. It is important to start the optimization time window at a time when the initial transient has left the domain and the local mesh resolution is adequate to resolve the weakened vortex. The optimization time window commences at time $t_{0}=15.75$ when the vortex is located at $(-2.63,0.67)$ and has circulation -0.075 . It should be noted that by this time the iso-vorticity lines in the domain look elliptic and not circular due to the induced velocity of the image vortex (see Figure 1).

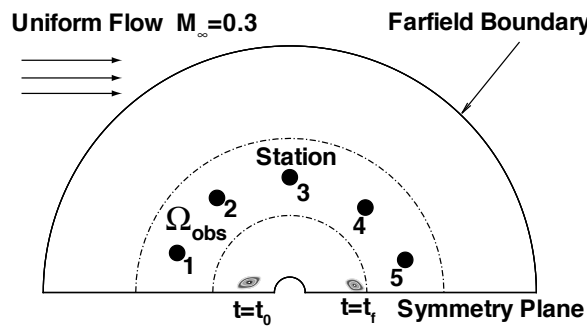

(a) case 1

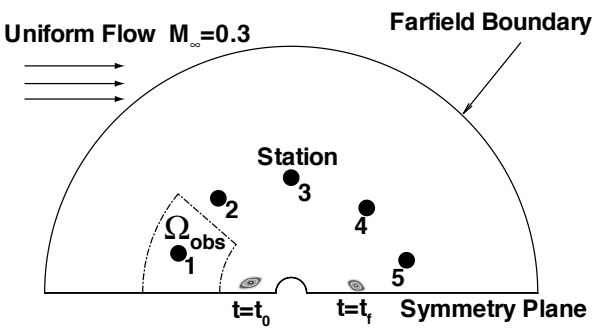

(b) case 2

Figure 1. Schematic of problem set-up.

The optimization time window consists of 1700 uniform time steps $\Delta t=0.009$ concluding at time $t_{f}=31.05$. The farfield boundary is 16 cylinder radii away from the center of the cylinder. The mesh has 128 uniformly distributed points in the azimuthal direction and 256 points in the radial direction. The grid point distribution in the radial direction is nonuniform. The mesh spacing at the wall is 0.04 , which remains almost constant for $r \in[1,10]$, increasing exponentially for $r \in[10,16]$.

Results are presented for two optimal control problems referred to as case 1 and case 2. In case 1, shown in Figure 1(a), the observation region is defined over the annular region between two concentric semi-circles of radii 5 and 10 resolved with 125 mesh points in the radial direction and the control, $g$, is allowed to be nonzero everywhere on the cylinder surface. In case 2, shown in Figure 1(b), the observation region is a subset of the annular region used in case 1 defined by a $45^{\circ}$ wedge, measured clockwise from the leading edge, and control is nonzero only on a quarter of the cylinder surface measured from the leading edge. For both cases, the time history of pressure fluctuations is recorded at five stations at radius 7.5. The stations 1-5 are separated by $30^{\circ}$ in the azimuthal direction measured from the leading edge. 


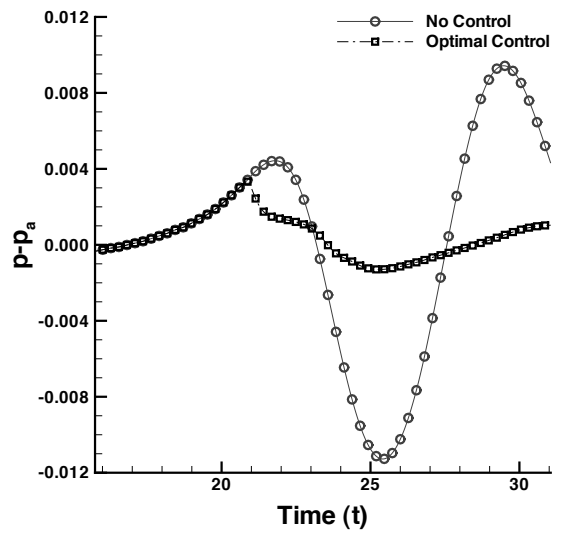

(a) Station 1: $\theta=30^{\circ}$

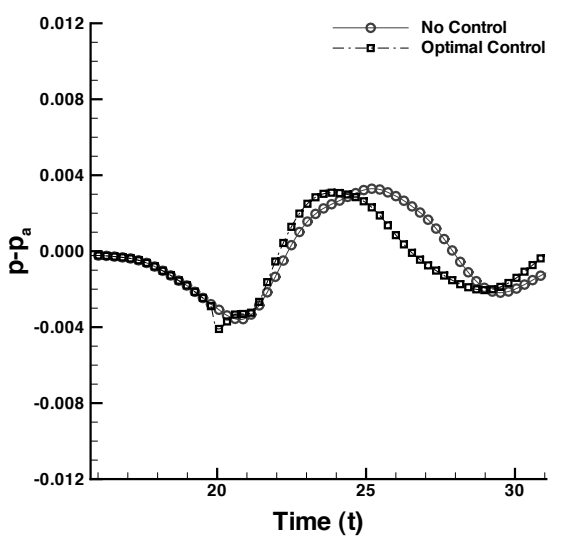

(c) Station 3: $\theta=90^{\circ}$

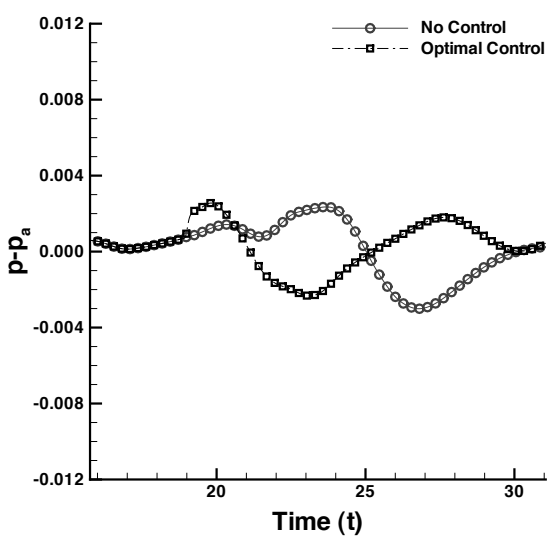

(e) Station 5: $\theta=150^{\circ}$

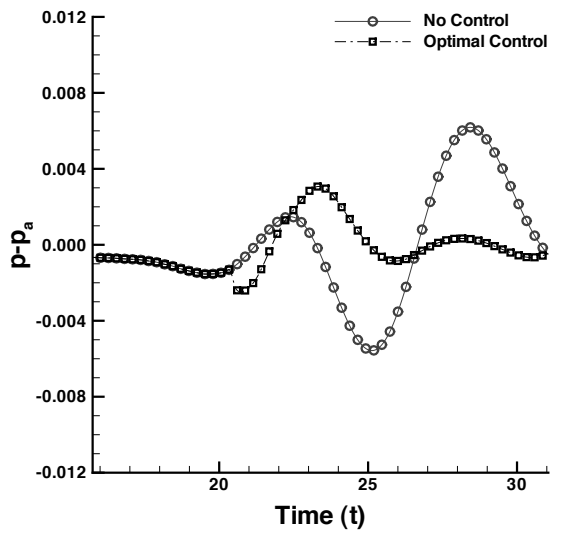

(b) Station 2: $\theta=60^{\circ}$

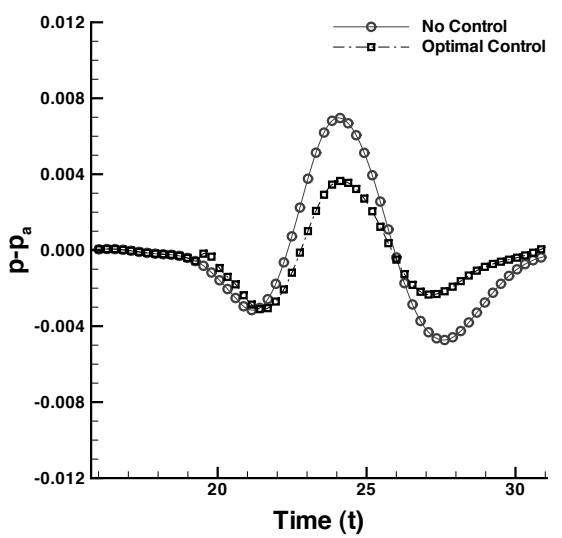

(d) Station 4: $\theta=120^{\circ}$

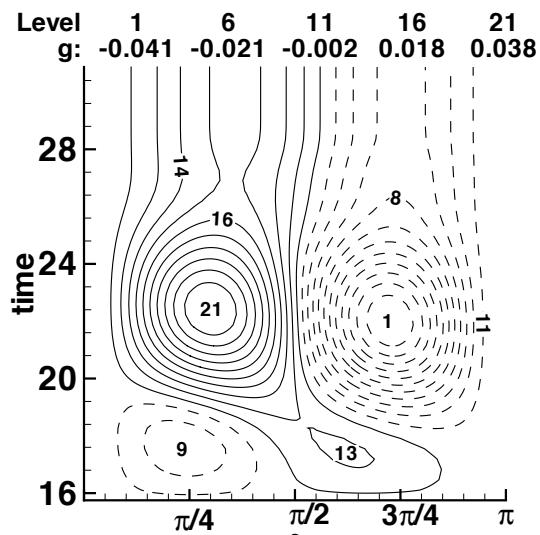

(f) Optimal Control Distribution

Figure 2. Case 1: Time history of pressure at stations 1-5 and contours of control $g$ versus time and position on the surface. 
Before proceeding with a detailed discussion of the results, we first mention the steps that we took to verify that the mesh resolution and domain size stated above are adequate. We performed a limited number of calculations on a mesh with twice the resolution and found that the uncontrolled BVI acoustic amplitude was about $11 \%$ greater on the finer mesh and that this difference was likely due to differences in the transient phase of the simulation prior to the start of optimal control. The optimal controls computed on the baseline mesh gave almost exactly the same reduction in acoustic intensity when used on the finer mesh indicating that the optimal controls are well predicted on the baseline mesh. Likewise, we also performed a calculation on a domain where the farfield radius was doubled. Here the uncontrolled acoustic amplitude was within $3 \%$ of the value on the baseline mesh. Again, the optimal controls computed on the baseline mesh gave nearly identical reduction in the objective functional on the larger mesh indicating that our farfield boundary treatment performs well. Based on these studies, we judge the numerical resolution and domain size to be adequate and move on to the presentation of results.

\subsection{Uncontrolled Flow}

Pressure fluctuations for the uncontrolled flow are shown in Figure 2(a-e). This figure also shows optimal control results that will be discussed in the following section. Focusing on the uncontrolled flow, we see that at station 1, the pressure fluctuation is very typical of a BVI event. At the other stations, the BVI acoustic amplitude is significantly less than at station 1 indicating that the strongest acoustic amplitude is generated $30^{\circ}$ from the horizontal which is consistent with prior BVI investigations.

\subsection{Optimal control: case 1}

In our first control case, we decided to utilize the full potential of optimal control theory by considering an objective function that targets the BVI sound signature over the entire cylinder and where the control is allowed to vary over nearly all of the cylinder surface. The wall-normal velocity control can be actuated on the cylinder surface between angles $(4 \pi / 127,123 \pi / 127)$ measured clockwise from the negative $x$ axis, and the observation region, $\Omega_{\text {obs }}$, is the whole annular region $r \in[5,10] ; \theta \in[0$, $\pi$ ]. The regularization coefficients in [9] are set to $\alpha_{0}=1, \alpha_{1}=\alpha_{2} \ldots \alpha_{4}=10^{-8}$, to allow for generous actuation of the control. The optimization is started with zero control and the initial value of the objective functional is $J_{0}=0.0426$. The optimization process is terminated after 15 nonlinear conjugate-gradient iterations and $J$ is reduced to 0.0102 , slightly less than a quarter of its starting value, and the modulus of the gradient of the objective functional has been reduced from $\left\|\nabla J_{0}\right\|=1.038$ to $\left\|\nabla J_{\text {final }}\right\|=$ 0.045. The contribution of the regularization terms to the final value of the objective functional is negligible. Thus, the change in the objective functional directly indicates the change in the acoustic intensity in the observation region, and the optimal control achieves a $6.2 \mathrm{db}$ reduction in acoustic intensity in the observation region.

We now return to Figure 2(a-e) and compare the pressure fluctuations at each of the sensing stations for the no-control and controlled flow. Station 1, which had the largest BVI sound for the uncontrolled flow, shows the most dramatic change with optimal 
control. The strong BVI noise at this station has been significantly reduced by the control. The sound at stations 2 and 4 is also noticeably reduced, while that at stations 3 and 5 is only slight reduced. Notice, however, that the acoustic pulse at station 5 for the controlled flow is out of phase with the uncontrolled flow. Because information travels at the speed of sound relative to the fluid, one observes a delay between the start of control at $t_{0}=15.75$ and any corresponding change at the measurement stations. For example, at station 1 , the effect of the control is not felt until $t \approx 21$.

The space-time distribution of control on the cylinder surface is shown in Figure 2(f). After the first 4 time units, we see that over the front half of the cylinder the control is primarily blowing while suction is applied to the rear half of the cylinder. The peak magnitudes of suction and blowing are roughly equal at $4 \%$ of the freestream velocity. The work performed by the control is estimated by assuming that fluid is available for control actuation at the local mean flow conditions and integrating the flux of enthalpy added to the flow across the controlled boundary $\Gamma_{c}$ during the time interval $\left[t_{0}, t_{f}\right]$. The ratio of this estimate of the work performed by the control to the kinetic energy in the vortex is $18 \%$ which indicates that the control input is small compared to the energy containing dynamics of the flow. For another measure of the efficiency of the optimal control, we compute the ratio of the maximum acoustic power input due to the boundary control to the maximum acoustic power input due to the BVI acoustic source (the interaction of the mean flow and the vortex). These terms are computed based on equation (1.87) of Goldstein (1976) and the ratio is found to be $21 \%$ indicating that the peak acoustic power input by the control is significantly less than the peak acoustic power of the BVI source term.

The vortex trajectories for the no-control and optimal control simulations are shown in Figure 3. It is interesting to note that the control does not appear to alter the path of the vortex whose interaction with the potential mean flow over the solid surface is the source of noise production. While this is not a particularly sensitive measure of the effect of the control on the vortex, it does suggest that the optimal control does not significantly change the vortex miss-distance as its means of reducing BVI noise.

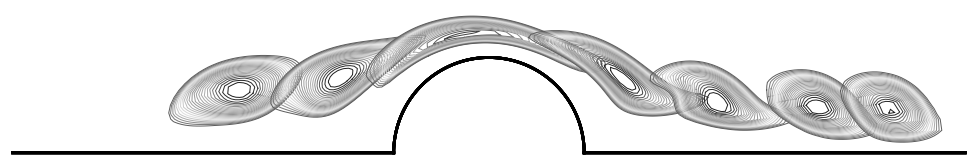

(a) no control

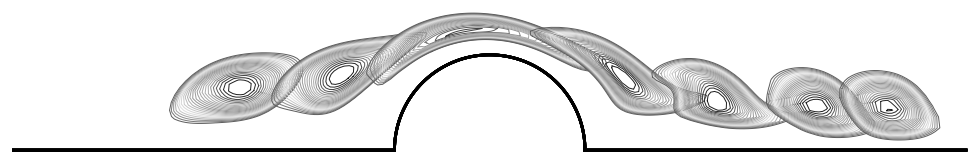

(b) optimal control

Figure 3. Vortex trajectories. 


\subsection{Optimal control: case 2}

Since the BVI noise in the uncontrolled flow is strongest at $30^{\circ}$ from the negative $x$ axis and our first optimal control results found the most significant reduction in noise in this region, we decided to limit the observation region in our objective functional to focus on this area. Likewise, we also limit the region over which control can act to the front quarter of the half cylinder.

To make this precise, the observation region $\Omega_{\mathrm{obs}}$, is the $\pi / 4$ wedge, $r \in[5,10]$ and $\theta \in[0, \pi / 4]$, where $\theta$ is measured clock-wise from the negative $x$ - axis, and the wall-normal velocity control is only allowed between angles $[0, \pi / 4]$. Other parameters are identical to those of case 1 . The optimization is started with zero control and the initial value of the objective functional is $J_{0}=0.025089$. The optimization process is terminated after 7 nonlinear conjugate-gradient iterations when $J$ is reduced to 0.001351 , a significant reduction of about $94.6 \%$. The modulus of the gradient is also reduced by about two orders of magnitude from $\left\|\nabla J_{0}\right\|=$ 0.1055 to $\left\|\nabla J_{\text {final }}\right\|=0.0011$. Again, the contribution of the regularization terms to the final value of the objective functional is negligible so that the change in the objective functional indicates a $12.7 \mathrm{db}$ change in acoustic intensity in the observation region.

The time history of pressure fluctuations at the recording stations $1-5$ for both no-control and optimally controlled flows are shown in Figures 4(a-e). The first station is located in the observation region and the noise amplitude is reduced significantly from the time the effect of the control is felt at that station. However, stations 2-5 are outside the observation region and, unlike case 1 , the noise magnitude is increased on average at these stations. These plots clearly show that the control mechanism focuses on the observation region and attempts to minimize the sound pressure level in that region only, at the expense of other regions in the flow. Whether these increases in sound pressure amplitude outside the observation window are acceptable depends on the particular application. Of course, if an overall reduction in BVI sound at all angles is desired, then an observation region like that used in case 1 is more appropriate.

Contours of the control are shown in Figure 4(f). Similar to case 1 in the forward sector, the primary control actuation is blowing where the peak amplitude is now about $5.2 \%$ of the freestream velocity. The ratio of the work performed by the control to the kinetic energy in the vortex is only $5.3 \%$ which indicates that the control input is very small compared to the energy containing dynamics of the flow. As in case 1, we also compared the ratio of maximum acoustic power input by the control to the maximum acoustic power input by the BVI sound source and found the ratio to be $11.5 \%$ for this case. Similar to case 1, the vortex trajectory is not noticeably modified from that of the uncontrolled flow. 


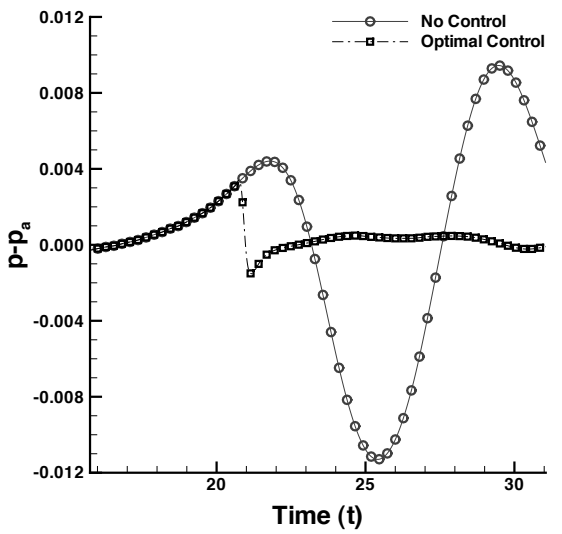

(a) Station 1: $\theta=30^{\circ}$

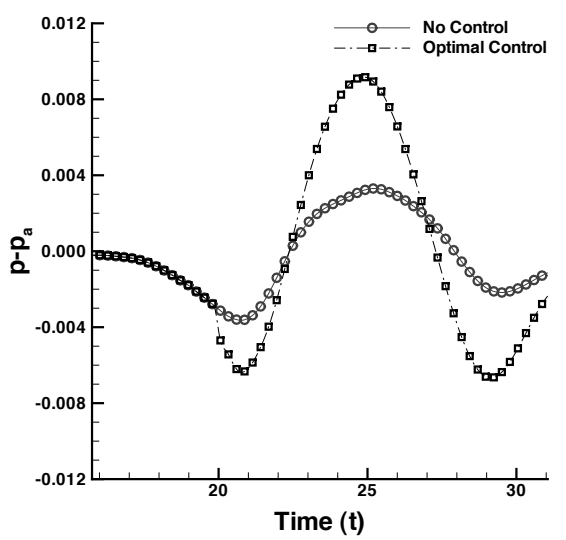

(c) Station 3: $\theta=90^{\circ}$

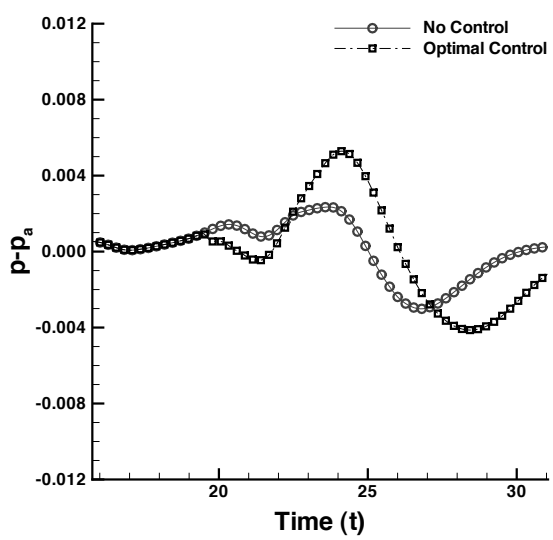

(e) Station 5: $\theta=150^{\circ}$

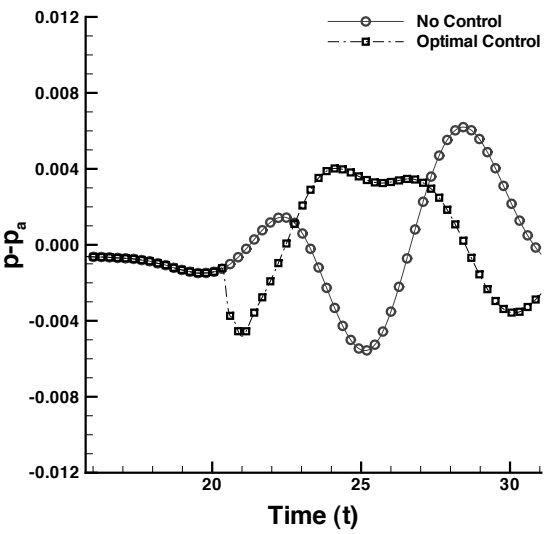

(b) Station 2: $\theta=60^{\circ}$

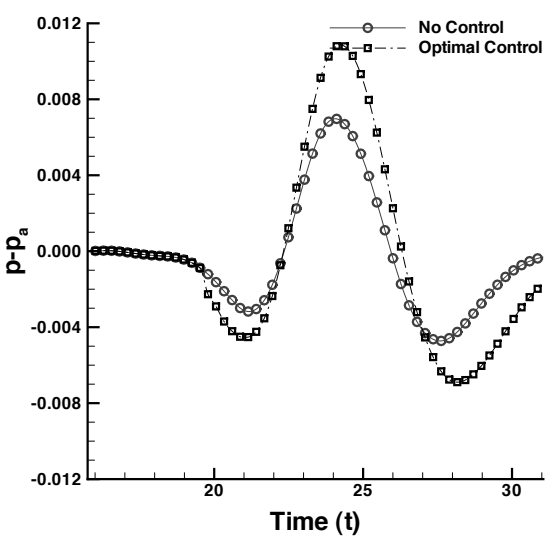

(d) Station 4: $\theta=120^{\circ}$

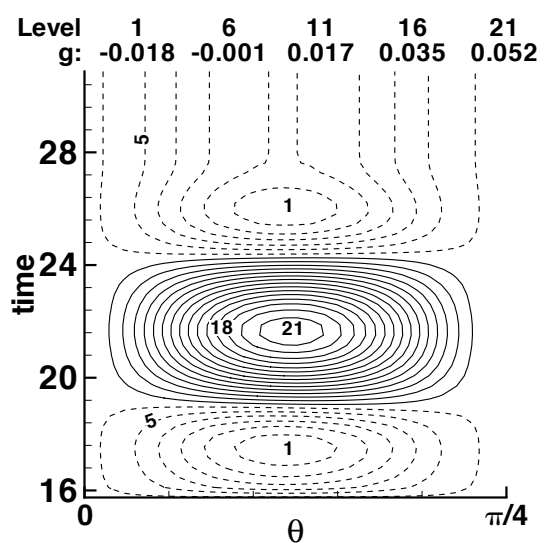

(f) Optimal Control Distribution

Figure 4. Case 2: Time history of pressure at stations 1-5 and contours of control $g$ versus time and position on the surface 


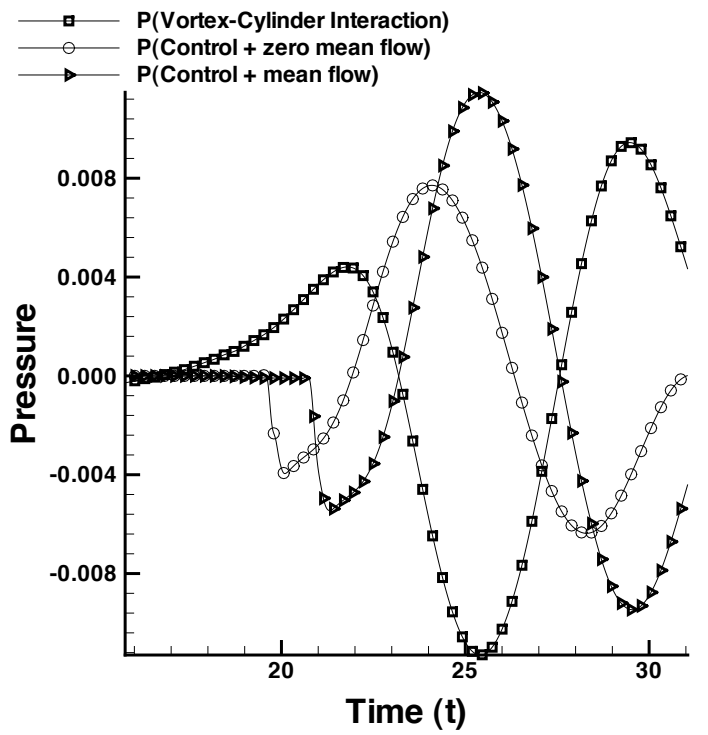

Figure 5. Time history of pressure fluctuations at station $1, \theta=30^{\circ}$.

\subsection{DISCUSSION}

In order to elucidate the underlying mechanism of noise reduction, the time history of pressure fluctuations at station 1 for case 2 are presented in Figure 5 for the following conditions: 1) no-control, 2) actuation of the optimal control distribution in a zeromean flow, and 3) actuation of the optimal control distribution in the mean flow over the cylinder without the superimposed vortex. Control actuation in zero-mean flow produces a wave packet whose amplitude is about $2 / 3$ of the observed BVI noise. The effect of its actuation is felt at an earlier time in the observation region because of the absence of the mean flow. Actuation of the optimal control in the mean flow without the vortex produces a wave packet that has virtually the same amplitude and is nearly perfectly out of phase with the BVI noise for $t \geq 22.5$. This clearly shows that the interaction of the mean potential flow and the control actuation at the boundary amplifies the generated wave-packet by almost a factor of 1.5 with respect to the zero flow case and provides the wave packet with the correct phase to cancel out the BVI noise. These tests also point out that for this model problem, the effect of the vortical field on the wave generated by the optimal control actuation is insignificant.

Thus, the optimal control uses its complete knowledge of the flow physics to generate a control distribution of wall transpiration that modulates the mean potential flow generating a wave packet that tends to cancel the BVI noise in the observation region.

During the course of this research, we became aware of two recent U.S. patents for on-blade BVI control devices that share similarities with our approach. The patent by Remington et al. (2001) is similar to our research in that they use arrays of suction and 
blowing panels on the rotor surface as their control actuator. In their configuration, suction panels are mounted near the leading edge that admit air into the interior of the rotor that is then expelled from panels near the trailing edge. The actuation of this system is based on noise measurements (perhaps with microphones on the tail boom, or surface pressure sensors on the rotor itself). Interestingly, our optimal control distributions primarily employ blowing near the leading edge with suction downstream (case 1) which is opposite to the setup described in this patent. They also utilize control jets at the blade tips that are actuated to accelerate the decay of the tip vortices. Based on these observations, it is unlikely that the mechanism for BVI noise mitigation is similar to our optimal controls.

In Hassan et al. (2000) one or more synthetic jet type actuators are mounted on the upper and lower surfaces of a rotor blade near the leading edge and are actuated based on measured surface pressure differential between the upper and lower surface in an attempt to counteract changes in surface pressure due to a BVI event. They present results from a computer simulation of BVI for flow over a NACA-0012 airfoil under conditions similar to those studied here (vortex strength 0.20 , core radius 0.25 chord, and miss distance 0.25 chord) and report a reduction in perturbation lift coefficient and thereby infer an attenuation in BVI noise. Interestingly, the peak jet velocity they use is $5 \%$ of the freestream which is very similar to that of our optimal controls. While insufficient information is provided in the patent to determine whether the reduction in actual BVI sound pressure levels is comparable to that obtained by our optimal controls, it is clear that the two approaches are similar and that one potential means of designing a practical system based on optimal control is through the use of synthetic jet type actuators. Likewise, our optimal control approach can be used to help guide and improve the design of such a system which is a promising direction for future research.

\section{CONCLUSIONS}

This work focuses on the use of on-blade normal velocity actuation for controlling the impulsive noise arising from blade-vortex interaction (BVI). The problem is cast as a minimization problem where the objective function is the square of pressure deviation from the meanflow distribution in an observation region within a time window encompassing the interaction. A continuous adjoint gradient-based method is used to solve the proposed optimization problem. This study shows that optimal distributions of wall-normal suction and blowing can reduce BVI noise significantly. When the objective function targets the full BVI sound signature an overall $6 \mathrm{db}$ reduction in BVI noise is obtained. When the objective function is set to target only the forward scattered BVI sound, which dominates the BVI sound signature, then a $13 \mathrm{db}$ reduction in BVI noise is obtained in this region although the noise in other directions is increased. In all cases, the vortex trajectory remains unaltered, indicating that the optimal control actuation does not alter the interaction of the vortical and potential fields, which is the source of the BVI noise. Instead, the optimal control uses its complete knowledge of the flow physics to generate a control distribution of wall transpiration that modulates the mean potential flow generating a wave packet that tends to cancel the BVI noise in 
the observation region. This result clearly illustrates the ability of the optimal control approach in extracting information from the governing equations of motion for flow modification purposes and our future work will apply this technique to progressively more complex aeroacoustic control problems.

\section{ACKNOWLEDGEMENTS}

The work of S. Collis, K. Ghayour and M. Heinkenschloss was supported by Texas ATP grant 003604-0001, 1999. Computations were performed on an SGI Origin 2000 which was purchased with the aid of NSF SCREMS grant 98-72009.

\section{REFERENCES}

Abergel, F. and Temam, R. (1990). On some control problems in fluid mechanics. Theoretical and Computational Fluid Dynamics, Vol. 1, pp. 303-325.

Baeder, J. D. (1997). Passive design for isolated blade-vortex interaction noise reduction. In Proceedings of the 53rd Annual Forum of the American Helicopter Society, Part 1.

Berggren, M. (1998). Numerical solution of a flow-control problem: Vorticity reduction by dynamic boundary action. SIAM J. Scientific Computing, Vol. 19, pp. 829860.

Caradonna, F. X., Strawn, R., and Bridgeman, J. O. (1988). An experimental and computational study of rotor-vortex interactions. Vertica, Vol. 12, No. 4.

Carpenter, M. H., Gottlieb, D., and Abarbanel, S. (1993). Stable and accurate boundary treatments for compact, high-order finite difference schemes. Appl. Num. Math., Vol. 12, No. 1-3, pp. 55-87.

Collis, S. S. (1997). A Computational Investigation of Receptivity in High-Speed Flow Near a Swept Leading-Edge. PhD thesis, Stanford University.

Collis, S. S., Ghayour, K., Heinkenschloss, M., Ulbrich, M., and Ulbrich, S. (2001a). Numerical solution of optimal control problems governed by the compressible Navier-Stokes equations. In Hoffmann, K.-H., Lasiecka, I., Leugering, G., Sprekels, J., and Tröltzsch, F., editors, Proceedings of the International Conference on Optimal Control of Complex Structures, International Series of Numerical Mathematics, Vol. 139, pp. 43-55. Birkhäuser Verlag.

Collis, S. S., Ghayour, K., Heinkenschloss, M., Ulbrich, M., and Ulbrich, S. (2001b). Towards adjoint-based methods for aeroacoustic control. AIAA Paper 2001-0821.

Collis, S. S., Ghayour, K., and Heinkenschloss, M. (2002). Optimal control of aeroacoustic flows: Transpiration boundary control. AIAA 2002-2575.

Fursikov, A. V., Gunzburger, M. D., and Hou, L. S. (1998). Boundary value problems and optimal boundary control for the Navier-Stokes systems: The two-dimensional case. SIAM J. Control Optimization, Vol. 36, No. 852-894.

Gad-el-Hak, M. (2000). Flow Control. Passive, Active, and Reactive Flow Management. Cambridge University Press, New York.

Gervais, M. (2001). Tiltrotor blade-vortex interaction (BVI) noise control through 
non-unique longitudinal force trim. Presented at the Southeast Lichten Award Regional Competition of the American Helicopter Society, College Park, Maryland, January 10, 2001. http://www.glue.umd.edu/ bsim/Publications.html.

Goldstein, M. E. (1976). Aeroacoustics. McGraw-Hill.

Gunzburger, M. D. and Manservisi, S. (2000). The velocity tracking problem for Navier-Stokes flows with boundary control. SIAM J. Control Optimization, Vol. 39, No. 594-634.

Hassan, A. A., Straub, F. K., and Domzalski, D. B. (2000). Oscillating air jets for helicopter aerodynamic control and BVI noise reduction. United States Patent 6,092,990, July 25, 2000. Assignee: McDonnell Douglas Helicopter Company (Meza, AZ). http://patft.uspto.gov/.

He, B., Ghattas, O., and Antaki, J. F. (1997). Computational strategies for shape optimization of time-dependent Navier-Stokes flows. Technical Report CMU-CML-97102, Carnegie Mellon University.

He, J.-W., Glowinski, R., Metcalfe, R., Nordlander, A., and Periaux, J. (2000). Active control and drag optimization for flow past a circular cylinder. I. oscillatory cylinder rotation. Journal of Computational Physics, Vol. 163, pp. 83-117.

Israeli, M. and Orszag, S. A. (1981). Approximation of radiation boundary condition. Journal of Computational Physics, Vol. 41, pp. 115-135.

JanakiRam, R. D. (1990). Aeroacoustics of rotorcraft. Technical Report FDP Special Course, AGARD.

Krishnamoorthy, S. and Marshall, J. S. (1998). Three-dimensional blade-vortex interaction in the strong vortex regime. Physics of Fluids, Vol. 10, No. 11, pp. 2828-2845.

Lee, S. (1994). Reduction of blade-vortex interaction noise through porous leading edge. AIAA Journal, Vol. 32, No. 3, pp. 480-488.

McCluer, M. S. (1996). Helicopter blade-vortex interaction noise with comparisons to CFD calculations. Technical Report NASA TM-110423, NASA Ames Research Center.

McMichael, J. M. (1996). Progress and prospects for active flow control using microfabricated electro-mechanical systems (MEMS). AIAA Paper 96-0306.

Nakamura, Y. (1981). Prediction of blade vortex interaction from measured blade pressure. AHS Seventh European Rotorcraft Powered Lift, 1981.

Nocedal, J. and Wright, S. J. (1999). Numerical Optimization. Springer Verlag, Berlin, Heidelberg, New York.

Remington, P. J., Coney, W. B., and Curtis, A. R. D. (2001). Use of flow injection and extraction to control blade vortex interaction and high speed impulsive noise in helicopters. United States Patent 6,283,406, September 4, 2001. Assignee: GTE Service Corporation (Irving, TX). http://patft.uspto.gov/.

Schmitz, F. H. (1998). Reduction of blade-vortex interaction (BVI) noise through Xforce control. Journal of the American Helicopter Society, Vol. 43, No. 1, pp. 14-24. 
Sesterhenn, J. (2001). A characteristic-type formulation of the Navier-Stokes equations for high order upwind schemes. Computers \& Fluids, Vol. 30, pp. 37-67.

Shu, C.-W. (1997). Esentially non-oscillatory and weighted essentially non-oscillatory schemes for hyperbolic conservation laws. Technical Report 97-65, ICASE.

Sim, B. W.-C. and Schmitz, F. H. (2000). Blade-vortex interaction (BVI) noise: Retreating side characteristics, sensitivity to chordwise loading and unsteady aerodynamics. Presented at the American Helicopter Society (AHS) Aeromechanics Specialists' Meeting, Atlanta, Georgia, November 13-14, 2000.

http://www.glue.umd.edu/ bsim/Publications.html.

Widnall, S. (1971). Helicopter noise due to blade-vortex interaction. Journal of the Acoustical Society of America, Vol. 50, pp. 354-365.

Yu, Y. H., Gmelin, B., Splettstoesser, W., Philippe, J. J., Prieur, J., and Brooks, T. F. (1997). Reduction of helicopter blade-vortex interaction noise by active rotor control technology. Progress in Aerospace Sciences, Vol. 33, No. 9-10, pp. 647-687. 